\title{
Disorder dependence of the ferromagnetic quantum phase transition
}

\author{
Y. Sang ${ }^{1}$, D. Belitz ${ }^{1,2}$ and T.R. Kirkpatrick ${ }^{3}$ \\ ${ }^{1}$ Department of Physics and Institute of Theoretical Science, University of Oregon, Eugene, OR 97403, USA \\ 2 Materials Science Institute, University of Oregon, Eugene, OR 97403, USA \\ 3 Institute for Physical Science and Technology, and Department of Physics, \\ University of Maryland, College Park, MD 20742, USA
}

(Dated: August 14, 2021)

\begin{abstract}
We quantitatively discuss the influence of quenched disorder on the ferromagnetic quantum phase transition in metals, using a theory that describes the coupling of the magnetization to gapless fermionic excitations. In clean systems, the transition is first order below a tricritical temperature $T_{\mathrm{tc}}$. Quenched disorder is predicted to suppress $T_{\mathrm{tc}}$ until it vanishes for residual resistivities $\rho_{0}$ on the order of several $\mu \Omega \mathrm{cm}$ for typical quantum ferromagnets. We discuss experiments that allow to distinguish the mechanism considered from other possible realizations of a first-order transition.
\end{abstract}

PACS numbers: 75.20.En; 75.30.Kz; 64.70.Tg; 05.30Rt

There is substantial experimental evidence for the quantum ferromagnetic transition in clean metals to be generically of first order. Examples of systems that were expected to display a quantum critical point, but instead display a first-order transition if the Curie temperature is suppressed, are $\mathrm{MnSi}$ [1, 2], $\mathrm{ZrZn}_{2}$ [3], $\mathrm{UGe}_{2}$ [4] 6], and URhGe [7. All of these are low-temperature ferromagnets (although the magnetic moment in some of them is not small) with Curie temperatures $T_{\mathrm{c}}$ between $\approx 10 \mathrm{~K}$ $(\mathrm{URhGe})$ and $\approx 50 \mathrm{~K}\left(\mathrm{UGe}_{2}\right)$, and magnetic moments per formula unit of about $0.17,0.4$, and $1.5 \mu_{\mathrm{B}}$ for $\mathrm{ZrZn}_{2}$, $\mathrm{MnSi}$ and URhGe, and $\mathrm{UGe}_{2}$, respectively. $T_{\mathrm{c}}$ is tunable by hydrostatic pressure or, for URhGe, by an external magnetic field transverse to the easy axis. A tricritical point separates a line of second-order transitions above the tricritical temperature $T_{\mathrm{tc}}(\approx 5 \mathrm{~K}, 10 \mathrm{~K}, 1 \mathrm{~K}$, and $24 \mathrm{~K}$ in $\mathrm{ZrZn}_{2}, \mathrm{MnSi}, \mathrm{URhGe}$, and $\mathrm{UGe}_{2}$, respectively) from a line of first-order transitions below, and in all of these materials tricritical wings have been observed in an external magnetic field. The respective values of the critical field at the wing tips are $H_{\mathrm{c}} \gtrsim 0.05 \mathrm{~T}, \approx 0.6 \mathrm{~T}, \approx 1 \mathrm{~T}$, and $\approx 10 \mathrm{~T}$. The qualitative phase diagram is shown in the rightmost panel in Fig. 1. Evidence for a first-order quantum phase transition (QPT) at low temperatures has been found in many other systems, but the phase diagram has not been mapped out completely, or the tricritical point is not accessible (as in $\mathrm{UCoAl}[8]$ ).

These observations are remarkable because of their universality. The only known instances in which the QPT to a homogeneous ferromagnet is not observed to be of first order are the quasi-1-d material $\mathrm{YbNi}_{4} \mathrm{P}_{2}$, 9], where the physics is expected to be quite different from that of true bulk metals, and various disordered materials where the transition is tuned by chemical composition, e.g., $\mathrm{URu}_{2-x} \mathrm{Re}_{x} \mathrm{Si}_{2}$ [10. The weakly disordered compounds $\mathrm{Ni}_{x} \mathrm{Pd}_{1-x}$ [11] and $\left(\mathrm{Cr}_{1-x} \mathrm{Fe}_{x}\right)_{2} \mathrm{~B}$ [12] we will come back to. Also remarkable is the stark disagreement between experiment and early theories. The quantum ferromagnetic transition as described by Stoner's mean-field the-

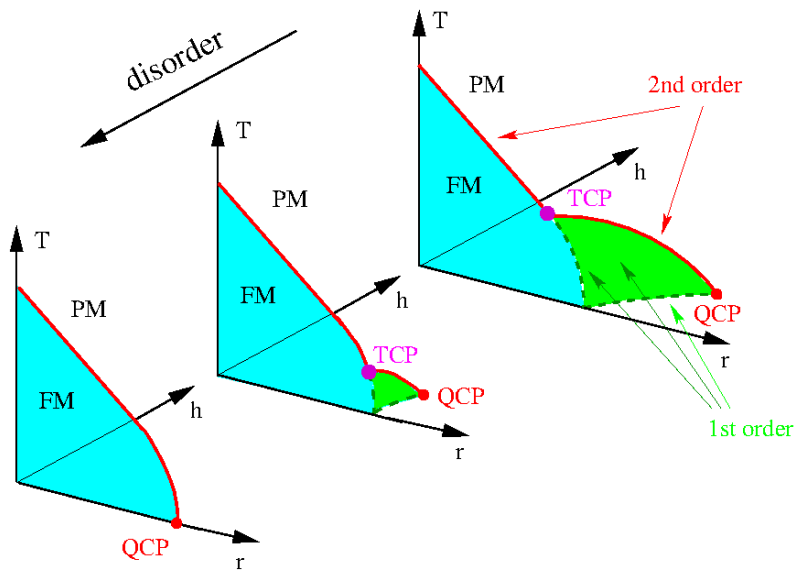

FIG. 1: (Color online) Evolution of the phase diagram of a metallic quantum ferromagnet in the space spanned by temperature $(T)$, magnetic field $(h)$, and the control parameter $(r)$ with increasing disorder. Shown are the ferromagnetic (FM) and paramagnetic (PM) phases, lines of second-order transitions (solid red), the tricritical point (TCP), and a surface of first-order transitions ("tricritical wing") that ends in a quantum critical point (QCP). With increasing disorder the tricritical temperature decreases, the wings shrink, and above a critical disorder strength a QCP is realized in zero field.

ory 13 is generically continuous. It was later considered as an example by Hertz in his seminal renormalizationgroup treatment of QPTs [14, which also predicted a continuous transition with mean-field critical behavior.

A theory that explains, and indeed predicted, the observed universality was developed in Refs. 15, 17, It relies on the coupling between the magnetization and soft or gapless fermionic excitations with a ballistic frequencymomentum relation that exist in any clean metal at $T=0$. It leads to an equation of state of the form

$$
h=r m-v m^{3} \ln \left(1 / m^{2}\right)+u m^{3},
$$

where $m$ is the magnetization in suitable units, $h$ is the external field, $r$ is the control parameter, and $v$ and $u$ are 
Landau parameters. The nonanalytic $m^{3} \ln (1 / m)$ term is the result of $m$ coupling to the soft modes in $d=3$; more generally its form is $m^{d}$. Crucially, $v>0$, which leads to a first-order transition at some $r>0$. This universal mechanism has been confirmed by a variety of other techniques [18 20]. $T>0$ gives the soft modes a mass, which cuts off the $\ln m$ nonanalyticity and leads to a tricritical point (rightmost panel in Fig. 1). Quenched disorder has two effects. First, it also cuts off the $\ln m$. Second, a coupling to diffusive soft modes leads to an $m^{d / 2}$ nonanalyticity whose sign is opposite of that of the nonanalytic term in the clean case. For sufficiently strong disorder, in $d=3$, one finds [21]

$$
h=r m+\frac{w}{\left(k_{\mathrm{F}} \ell\right)^{3 / 2}} m^{3 / 2}+u m^{3},
$$

with $w>0$ another parameter, $k_{\mathrm{F}}$ the Fermi wave number, which sets the microscopic length scale, and $\ell$ the elastic mean-free path. This leads to a continuous transition with non-mean-field exponents (leftmost panel in Fig. (1). Equations (1) and (2) both represent renormalized Landau theories, which replace the fluctuating orderparameter field by its mean. Since the order-parameter fluctuations at the QPT are above their upper critical dimension $\left(d_{\mathrm{c}}^{+}=1\right.$ (0) for the clean (disordered) case [14]), this should not affect the nature of the QPT. Indeed, in the disordered case a RG study has shown that orderparameter fluctuations leave the power laws described by Eq. (2) intact, although they lead to non-power-law modifications of the leading scaling behavior [22, 23].

While Eq. (1) is in qualitative agreement with all experiments on clean samples, there still are open questions. First, Eqs. (1) and (2) represent only the extremes of ultraclean and strongly disordered systems. Many experiments fall in between these two cases, e.g., $\mathrm{Ni}_{x} \mathrm{Pd}_{1-x}$, which shows a second-order transition with mean-field exponents at $x=0.027$ [11. Second, the mechanism proposed in Ref. 15 is not the only possibility for a firstorder transition; e.g., in classical compressible magnets the coupling between the magnetization and phonons can lead to a first-order transition [24]. This mechanism is not as universal as the one leading to Eq. (1), but Refs. 25-27 have argued that an adaptation to the quantum transition can explain the observations, at least in the case of the pressure-tuned quantum ferromagnets.

It is thus desirable to develop criteria that allow for a discrimination between the different theoretical ideas. In this Letter we show that the disorder dependence of the phase diagram allows for such a discrimination. Phonons are not qualitatively affected by disorder; therefore, if magnetostriction effects cause the first-order transition in a given material, then introducing disorder into the sample is expected to have only weak quantitative effects on the phase diagram. The mechanism of Ref. 15, on the other hand, is crucially affected by disorder, since in the strong-disorder limit the equation of state changes to Eq.
22. As we will show, our theory predicts three distinct disorder regimes. In a weak-disorder regime the transition is first order, but $T_{\mathrm{tc}}$ is gradually suppressed until it vanishes at a critical value of the disorder. For common quantum ferromagnets this is expected to happen for residual resistivities $\rho_{0}$ on the order of several $\mu \Omega \mathrm{cm}$. The resulting quantum critical point in an intermediatedisorder regime displays mean-field exponents consistent with Hertz theory in the observable critical region, although asymptotically close to the transition there will be a crossover to the non-mean-field critical behavior of Ref. 21. With increasing disorder the crossover moves away from the transition and becomes observable for values of $\rho_{0}$ on the order of tens of $\mu \Omega \mathrm{cm}$. Finally, in a strong-disorder regime with $\rho_{0}$ on the order of hundreds of $\mu \Omega \mathrm{cm}$ the non-mean-field critical behavior will be present in the entire critical region. However, for disorder that strong other effects may come into play. Our predictions can be tested by introducing quenched disorder, e.g., by means of irradiation, into any of the materials that display a first-order QPT, and following the changes in the phase diagram with increasing disorder.

To achieve these goals, we have constructed an equation of state that interpolates between Eqs. (1) and (2), and generalizes them to finite temperatures. We first state and discuss this equation of state, and then sketch its derivation. It takes the form

$$
\begin{aligned}
& h=r m+\frac{w}{\left(k_{\mathrm{F}} \ell\right)^{3 / 2}} m^{3 / 2} g\left(k_{\mathrm{F}} \ell m, c t / m\right) \\
& -v m^{3} \ln \left(\frac{1}{m^{2} / m_{0}^{2}+\left(\sigma_{0} / k_{\mathrm{F}} \ell+t\right)^{2}}\right)+u m^{3},
\end{aligned}
$$

which reduces to Eqs. (1), (2) in the limits $k_{\mathrm{F}} \ell \rightarrow \infty$, 0 . We will refer to the second and third term on the right-hand side as the diffusive and ballistic nonanalyticity, respectively. $m, h$, and $t$ are the dimensionless magnetization, magnetic field, and temperature, respectively, defined as follows. Let $\mu$ be the magnetization measured in $\mu_{\mathrm{B}}$ per volume, $H$ the external field, and $T$ the temperature. Let $n_{\mathrm{e}}$ be the conduction electron density, and $T_{\mathrm{F}}$ the Fermi temperature (or, more generally, the microscopic energy scale). Then $m=8 \mu / \pi n_{\mathrm{e}}$, $h=\mu_{\mathrm{B}} H / k_{\mathrm{B}} T_{\mathrm{F}}$, and $t=3 \pi T / T_{\mathrm{F}} . \quad v$ and $w$ depend on a coupling constant $\gamma_{\mathrm{t}}$ that measures the strength of conduction-electron correlations, with $\gamma_{\mathrm{t}} \ll 1$ and $\gamma_{\mathrm{t}}=O(1)$ corresponding to weakly and strongly correlated systems, respectively. Another coupling constant $c=O(1)$ describes the coupling between the magnetization and the conduction-electron spin density. In terms of $\gamma_{\mathrm{t}}$ and $c$ one finds, for small $\gamma_{\mathrm{t}}, v=c \gamma_{\mathrm{t}}^{4}$ and $w=c \gamma_{\mathrm{t}}$. $r \ll 1$ is the dimensionless control parameter for the transition, and $u=O(1)$ is a Landau parameter. $m_{0}$ and $\sigma_{0}$ set the scales for the magnetic moment and the disorder, respectively. In a simple model for itinerant ferromagnets one has $m_{0} \approx 7 / \gamma_{\mathrm{t}}$ and $\sigma_{0}=1$; more generally $m_{0}$ and $0.1 \lesssim \sigma_{0} \leq 1$ are independent microscopic scales that de- 
pend on the band structure and the correlation strength, see the discussion below. Finally,

$$
g(y, z)=\frac{1}{g_{0}} \int_{0}^{1 / y} d x \int_{z}^{\infty} d \omega \frac{\sqrt{x} \omega\left[2(x+\omega)^{2}+1\right]}{(x+\omega)^{3}\left[(x+\omega)^{2}+1\right]^{2}}
$$

with $g_{0}=\pi / 3 \sqrt{2} \approx 0.74$ is normalized such that $g(0,0)=$ 1. $g(y, 0)$ is well approximated by

$$
g(y, 0) \approx 1 /\left[1+y^{3 / 2} /\left(9 g_{0}+y / g_{0}\right)\right] .
$$

We now discuss typical values for the various parameters in Eq. (3a), initially for a clean system. With $k_{\mathrm{F}} \approx 1 \AA^{-1}$, and a formula unit volume of about $50 \AA^{3}$, we find a dimensionless saturation magnetization ranging from $m \approx 0.25$ for $\mathrm{ZrZn}_{2}$ to $m \approx 2.3$ for $\mathrm{UGe}_{2}$. Choosing $u=0.85, \gamma_{\mathrm{t}}=0.5$ (fairly strong correlation), and $c=1$, we have $v=0.06$. The tricritical temperature is $T_{\mathrm{tc}}=\left(T_{\mathrm{F}} / 3 \pi\right) e^{-u / 2 v}\left[15\right.$. With $T_{\mathrm{F}} \approx 10^{5} \mathrm{~K}$ we have $T_{\mathrm{tc}} \approx 10 \mathrm{~K}$, which is the correct order of magnitude for $\mathrm{ZrZn}_{2}, \mathrm{MnSi}$, and $\mathrm{UGe}_{2}$. A slightly lower value of $\gamma_{\mathrm{t}} \approx 0.45$ yields $T_{\mathrm{tc}} \approx 1 \mathrm{~K}$, as observed in URhGe. At the first-order transition at $T=0$, the magnetization changes discontinuously from zero to $m_{1}=m_{0} e^{-(1+u / v) / 2}$ [15]. For $m_{0}$ between 75 (for $\mathrm{ZrZn}_{2}$ ) and 350 (for $\mathrm{UGe}_{2}$ ), this yields $m_{1} \approx 0.05-0.25$, which is a reasonable fraction of the saturation magnetization in these materials. The critical field at the tips of the tricritical wings is given by $h_{c}=(4 / 3) e^{-13 / 4} m_{0}^{3} v e^{-3 u / 2 v}$ [16. With parameters as above this yields values from $H_{c} \approx 0.1 \mathrm{~T}$ to $H_{c} \approx 10 \mathrm{~T}$. This is again the correct order of magnitude compared with the experimental observations $2,3,5,5$.

Now consider quenched disorder. A Drude formula for $\rho_{0}$ with $k_{\mathrm{F}} \approx 1 \AA^{-1}$ yields $k_{\mathrm{F}} \ell \approx 1,000 \mu \Omega \mathrm{cm} / \rho_{0} . k_{\mathrm{F}} \ell$ thus ranges from $\gtrsim 10^{4}$ in a clean metal $\left(\rho_{0} \approx 0.1 \mu \Omega \mathrm{cm}\right)$ to about 10 in a poor metal $\left(\rho_{0} \approx 100 \mu \Omega \mathrm{cm}\right)$. This in turn implies that values of $k_{\mathrm{F}} \ell m$ between roughly 2.5 and $2 \times 10^{4}$ are realizable, with $m$ the saturation magnetization. With $m$ the actual magnetization, the lower limit is accordingly lower, depending on the minimal magnetization $m_{1}$ at the first-order transition, if any. From Eq. (3c) we see that $k_{\mathrm{F}} \ell m \approx 5$ is the demarcation between two different regimes, which falls well within this range.

All of the above, and everything that follows, are just rough order-of-magnitude estimates. With this in mind, we can distinguish the following regimes, classified according to the values of $k_{\mathrm{F}} \ell$ (clean vs. dirty samples) and $m$ (weak vs strong magnetism). They follow from the observation that the diffusive and ballistic nonanalycities, at $T=0$, are operative (inoperative) for $k_{\mathrm{F}} \ell m \lesssim 5$ ( and $k_{\mathrm{F}} \ell m \gtrsim m_{0} \sigma_{0}\left(k_{\mathrm{F}} \ell m \lesssim m_{0} \sigma_{0}\right)$, respectively.

Regime I (Clean/strong): $k_{\mathrm{F}} \ell m \gtrsim m_{0} \sigma_{0}$. The diffusive nonanalyticity is inoperative, the equation of state is given by Eq. (1), and the transition is first order with $m_{1}=m_{0} e^{-(1+u / v) / 2} \leq m$. For consistency, we must have $k_{\mathrm{F}} \ell m_{1} \gtrsim m_{0} \sigma_{0}$. With $u$ and $v$ as above, and $\sigma_{0} \approx 1 / 5$, this yields $k_{\mathrm{F}} \ell \gtrsim 300$, or $\rho_{0} \approx$ several $\mu \Omega \mathrm{cm}$.
Regime IIa (Intermediate): $5 \lesssim k_{\mathrm{F}} \ell m \lesssim m_{0} \sigma_{0}$. In this transient regime both nonanalyticities are inoperative, and the transition appears continuous with meanfield exponents in a range of $m$-values. However, as $m$ decreases, the system eventually enters Regime IIb or III.

Regime IIb (Intermediate): $k_{\mathrm{F}} \ell m \lesssim 5$ and $k_{\mathrm{F}} \ell \gtrsim$ $\left(k_{\mathrm{F}} \ell\right)^{*}$, with $\left(k_{\mathrm{F}} \ell\right)^{*}$ defined below. The ballistic nonanalyticity is inoperative, the equation of state is given by Eq. (2), and the transition is second order with the asymptotic critical behavior characterized by the nonmean-field exponents of Ref. 21. However, farther away from the transition this behavior will cross over to ordinary mean-field behavior at a disorder-dependent value $r^{*}$ of $r$. The crossover occurs when the last two terms in Eq. (2) are about equal. Having the crossover occur at $r=r^{*}$ thus requires a disorder given by $k_{\mathrm{F}} \ell=k_{\mathrm{F}} \ell^{*}=$ $w^{2 / 3} / u^{1 / 6}\left|r^{*}\right|^{1 / 2}$. If we require $r^{*}=0.01$ and choose $\gamma_{\mathrm{t}}=0.5$ and $u=1$ as before, we have $k_{\mathrm{F}} \ell^{*} \approx 6$, or $\rho_{0}^{*} \approx 150 \mu \Omega \mathrm{cm} . \rho_{0}^{*}$ is the disorder that separates Regime $\mathrm{IIb}$, where the transition is continuous with effectively mean-field exponents, from Regime III. Note that $\rho_{0}^{*}$ depends on the correlation strength via $w$; for $\gamma_{\mathrm{t}}=0.1$ (weak correlation) one has $\rho_{0}^{*} \approx 500 \mu \Omega \mathrm{cm}$.

Regime III (Dirty/weak): $k_{\mathrm{F}} \ell m \lesssim 5$ and $k_{\mathrm{F}} \ell \lesssim$ $\left(k_{\mathrm{F}} \ell\right)^{*}$. The equation of state is dominated by the diffusive nonanalyticity, and the transition is continuous with non-mean-field critical exponents in the entire critical region. This requires $\rho_{0}>\rho_{0}^{*}$, with $\rho_{0}^{*}$ ranging from approximately $100 \mu \Omega \mathrm{cm}$ for strongly correlated materials to hundreds of $\mu \Omega \mathrm{cm}$ for weakly correlated ones.

At a nonzero temperature, we see from Eq. (3a) that a disorder resulting in $k_{\mathrm{F}} \ell=\sigma_{0} T_{\mathrm{F}} / 3 \pi T_{\mathrm{tc}}$ has the same effect as $T=T_{\mathrm{tc}}$ in a clean system. That is, $\rho_{0} \gtrsim$ $10^{4} T_{\mathrm{tc}} / \sigma_{0} T_{\mathrm{F}} \approx$ several $\mu \Omega \mathrm{cm}$ will suppress $T_{\mathrm{tc}}$ to zero, consistent with the above estimate for the destruction of the first-order transition at $T=0$. The tricritical wings shrink, and eventually disappear, commensurate with the suppression of $T_{\mathrm{tc}}$. This prediction for the evolution of the phase diagram is shown in Fig. 1.

We now have the following predictions for the effects of quenched disorder on typical strongly correlated quantum ferromagnets: Disorder decreases $T_{\mathrm{tc}}$, and suppresses it altogether for a residual resistivity $\rho_{0}$ on the order of several $\mu \Omega \mathrm{cm}$. For larger $\rho_{0}$ the QPT will be continuous and appear mean-field-like in a substantial disorder range, $\rho_{0} \lesssim 100 \mu \Omega \mathrm{cm}$, with a crossover to non-meanfield behavior only extremely close to the transition. For even larger $\rho_{0}$ the critical behavior is characterized by the non-mean-field exponents of Refs. 21 23. However, for disorder that strong quantum Griffiths effects are expected to be present and compete with the critical behavior [28; to distinguish between the two one needs to measure the critical behavior of the magnetization. We stress that these predictions are semi-quantitative in nature. The important point is the existence of the three 
regimes; the disorder strengths that delineate them are expected to show substantial variations from material to material. We also note that quenched disorder can suppress a tricritical point in a purely classical model [29]. This mechanism is not dependent on the presence of conduction electrons and is expected to be characterized by different disorder scales than the one discussed here.

Some experimental evidence exists in favor of this scenario. For $\mathrm{ZrZn}_{2}$, the QPT was initially found to be second order [30, but with increasing sample quality a first-order transition emerged [3]. For $\mathrm{UGe}_{2}$, measured values of $T_{\mathrm{tc}}$ range from $24 \mathrm{~K}$ [5] to $31 \mathrm{~K}$ [7, which is possibly related to the sample quality, and in URhGe higher $T_{\mathrm{tc}}$ values were found for cleaner samples [31. All of these materials are strongly correlated as evidenced by their unusual electronic properties independent of the quantum magnetism. Finally, the observation of a quantum critical point with mean-field exponents in $\mathrm{Ni}_{x} \mathrm{Pd}_{1-x}$ 11], and possibly in $\left(\mathrm{Cr}_{1-x} \mathrm{Fe}_{x}\right)_{2} \mathrm{~B}$ [12, where the transition occurs at a small value of $x$, can be understood if one realizes that these system are likely in the intermediate Regime II. While these observations are encouraging, no systematic experimental study of the influence of quenched disorder on the phase diagram of quantum ferromagnets exists. Such an experiment would allow to discriminate between the explanation of the first-order transition discussed above and alternative proposals that predict only a weak disorder dependence of $T_{\mathrm{tc}}$.

We now sketch the derivation of Eq. (3a). The relevant soft fermionic modes, as functions of wave vector $\boldsymbol{k}$ and bosonic Matsubara frequency $\Omega_{n}$, are diffusive for disordered electrons and ballistic for clean ones [32],

$$
\mathcal{D}_{\text {diff }}=\frac{1}{\Omega_{n}+D \boldsymbol{k}^{2}} \quad, \quad \mathcal{D}_{\text {ball }}=\frac{1}{\Omega_{n}+v_{\mathrm{F}}|\boldsymbol{k}|}
$$

with $D=v_{\mathrm{F}}^{2} \tau / 3$ the diffusion coefficient, $\tau$ the elastic mean-free time, and $v_{\mathrm{F}}$ the Fermi velocity. The soft-mode propagator $\mathcal{D}$ can be modeled by $\mathcal{D}_{\text {diff }}$ for $|\boldsymbol{k}|<1 / \ell$, and by $\mathcal{D}_{\text {ball }}$ for $|\boldsymbol{k}|>1 / \ell$, with $\ell=v_{\mathrm{F}} \tau$ the elastic mean-free path. The magnetization $m$ couples to the soft fluctuations and cuts off the singularities that result from integrating over $\mathcal{D}$, which leads to nonanalytic dependences on $m$. Integrating out the soft modes yields a fluctuation correction to the free energy density $f$ of the form 32 .

$$
\Delta f=\frac{2}{V} \sum_{\boldsymbol{k}} T \sum_{n=1}^{\infty} \ln N\left(\boldsymbol{k}, \Omega_{n} ; m\right) .
$$

At $T=0$ the sum over $\Omega_{n}$ turns into an integral over a continuous variable $\omega$, and the effect of a nonzero temperature can be modeled by the replacement $\omega \rightarrow \omega+2 \pi T$. The fluctuation contribution to the equation of state is obtained by differentiating $\Delta f$ with respect to $m$. We measure $m$ in units of the conduction electron density $n_{\mathrm{e}}$, and the magnetic field $h$ in units of $k_{\mathrm{B}} T_{\mathrm{F}} / \mu_{\mathrm{B}}$. The Landau parameters $r$ and $u$ are then dimensionless. Up to factors of $O(1)$, the resulting equation of state takes the form of Eq. (3a) with $m_{0} \approx 7 / \gamma_{\mathrm{t}}$ and $\sigma_{0}=1$. These two values are based on a nearly-free-electron model for the conduction electrons. For real materials, $m_{0}$ is expected to be an independent parameter that depends on microscopic details. It sets the scale for the magnetic moment, which differs by a factor of 10 between, e.g., $\mathrm{ZrZn}_{2}$ and $\mathrm{UGe}_{2} . \sigma_{0}$ in general depends on the correlation strength and is $\leq 1$. The reason is that in a strongly correlated material two electrons with opposite spins cannot simultaneously take advantage of a disorder-induced potential well, because of the strong repulsion between the electrons. This is consistent with the fact that, in the absence of symmetry-breaking fields, interactions cause the disorder to get renormalized downward 33, 34. Correlations will thus effectively weaken the effects of the disorder; values of $\sigma_{0}$ between 1 (no correlation) and 0.1 (strong correlation) are reasonable based on the RG flow equations of Ref. 33. Finally, the soft-mode effects are stronger the lower the dimension; in $d=2$, the $m^{3} \ln (1 / m)$ term in Eq. (3a) turns into an $m^{2}$ term. For the diffusive modes, $d=2$ is the lower critical dimension, and the effects of quenched disorder become strong and very complex [34].

We thank Greg Stewart, Andrew Huxley, and Nihat Berker for discussions. This work was supported by the NSF under grant Nos. DMR-09-29966 and DMR09-01907. Part of this work was performed at the Aspen Center for Physics and supported by the NSF under Grant No. PHYS-1066293.

[1] C. Pfleiderer, G. J. McMullan, S. R. Julian, and G. G. Lonzarich, Phys. Rev. B 55, 8330 (1997). MnSi is a weak helimagnet, but is well approximated by a ferromagnet on length scales shorter than the helical wavelength.

[2] C. Pfleiderer, S. R. Julian, and G. G. Lonzarich, Nature (London) 414, 427 (2001).

[3] M. Uhlarz, C. Pfleiderer, and S. M. Hayden, Phys. Rev. Lett. 93, 256404 (2004).

[4] A. Huxley, I. Sheikin, E. Ressouche, N. Kernavanois, D. Braithwaite, R. Calemczuk, and J. Flouquet, Phys. Rev. B 63, 144519 (2001).

[5] V. Taufour, D. Aoki, G. Knebel, and J. Flouquet, Phys. Rev. Lett. 105, 217201 (2010).

[6] H. Kotegawa, V. Taufour, D. Aoki, G. Knebel, and J. Flouquet, J. Phys. Soc. Japan 80, 083703 (2011).

[7] A. Huxley, S. J. C. Yates, F. Lévy, and I. Sheikin, J. Phys. Soc. Japan 76, 051011 (2007).

[8] D. Aoki, T. Combier, V. Taufour, T. D. Matsuda, G. Knebel, H. Kotegawa, and J. Flouquet, J. Phys. Soc. Japan 80, 094711 (2011).

[9] C. Krellner, S. Lausberg, A. Steppke, M. Brando, L. Pedrero, H. Pfau, S. Tencè, H. Rosner, F. Steglich, and C. Geibel, New Journal of Physics 13, 103014 (2011).

[10] N. P. Butch and M. B. Maple, Phys. Rev. Lett. 103, 076404 (2009).

[11] M. Nicklas, M. Brando, G. Knebel, F. Mayr, W. Trinkl, 
and A. Loidl, Phys. Rev. Lett. 82, 4268 (1999).

[12] L. Schoop, M. Hirschberger, J. Tao, C. Felser, N. P. Ong, and R. J. Cava, arXiv:1406.4527 (2014).

[13] E. C. Stoner, Proc. Roy. Soc. London A 165, 372 (1938).

[14] J. Hertz, Phys. Rev. B 14, 1165 (1976).

[15] D. Belitz, T. R. Kirkpatrick, and T. Vojta, Phys. Rev. Lett. 82, 4707 (1999).

[16] D. Belitz, T. R. Kirkpatrick, and J. Rollbühler, Phys. Rev. Lett. 94, 247205 (2005).

[17] T. R. Kirkpatrick and D. Belitz, Phys. Rev. B 85, 134451 (2012).

[18] A. V. Chubukov, C. Pépin, and J. Rech, Phys. Rev. Lett. 92, 147003 (2004).

[19] U. Karahasanovic, F. Krüger, and A. G. Green, Phys. Rev. B 85, 165111 (2012).

[20] Our discussion is restricted to transitions from a paramagnet to a homogeneous ferromaget. The formation of inhomogeneous phases has been suggested in Refs. [18, 19, 35], among others.

[21] T. R. Kirkpatrick and D. Belitz, Phys. Rev. B 53, 14364 (1996).

[22] D. Belitz, T. R. Kirkpatrick, M. T. Mercaldo, and S. Sessions, Phys. Rev. B 63, 174427 (2001).

[23] D. Belitz, T. R. Kirkpatrick, M. T. Mercaldo, and S. Sessions, Phys. Rev. B 63, 174428 (2001).
[24] A. Aharony, in Phase Transitions and Critical Phenomena, edited by C. Domb and M. S. Green (Academic, New York, 1976), vol. 6, p. 358.

[25] G. A. Gehring, EPL 82, 60004 (2008).

[26] G. A. Gehring and M. R. Ahmed, J. Appl. Phys. 107, 09E125 (2010).

[27] V. Mineev, Comptes Rendus Physique 12, 567 (2011).

[28] T. Vojta, J. Low Temp. Phys. 161, 299 (2010).

[29] A. Falicov and A. N. Berker, Phys. Rev. Lett. 76, 4380 (1996).

[30] M. Grosche, C. Pfleiderer, G. J. McMullan, G. G. Lonzarich, and N. R. Bernhoeft, Physica B 206-207, 20 (1995).

[31] A. Huxley, private communication.

[32] D. Belitz, T. R. Kirkpatrick, and T. Vojta, Rev. Mod. Phys. 77, 579 (2005). Eq. 4a gives a simplified form of $\mathcal{D}_{\text {ball }}$ with the correct scaling properties.

[33] A. M. Finkelstein, Zh. Eksp. Teor. Fiz. 84, 168 (1983), [Sov. Phys. JETP 57, 97 (1983)].

[34] D. Belitz and T. R. Kirkpatrick, Rev. Mod. Phys. 66, 261 (1994).

[35] D. Belitz, T. R. Kirkpatrick, and T. Vojta, Phys. Rev. B 55, 9452 (1997). 